\title{
Rationalität der Justiz Output reicht nicht aus - Outcome ist das zentrale Thema
}

\author{
Bernd Maelicke
}

Heft 1 / 2004 der Neuen Kriminalpolitik war ein Genuss für den Leser, der sich über Zukunfts- und Ressourcenfragen einer rationalen Kriminalpolitik informieren wollte. Nahezu alle Beiträge beschäftigten sich mit der Fragestellung, wie der Einsatz der gesetzlichen, personalen, sachlichen und organisatorischen Mittel so gestaltet werden kann, dass ein Höchstmass an Effizienz und Effektivität erreicht werden kann. Diese neue Rationalität verändert offensichtlich in einem bisher unvorstellbaren Ausmaß das Denken und Handeln nicht nur der Justizpolitiker sondern auch der steuernden Ministerien, der Gerichtspräsidenten und der leitenden Staatsanwälte wie auch der JVA-Leiter. Outputorientierte Steuerung, Court Management, New Public Management - dies sind die neuen Paradigmen, die seit Anfang der 90er Jahre in allen staatlichen Organisationen zu grundlegenden Veränderungsprozessen geführt haben.

Am weitesten fortgeschritten sind diese Systemumstellungen in den Organisationen, die auf den Grundlagen der Kosten- und Leistungsrechnung bugetiert worden sind. Hier bekommen die Leitungskräfte über ein differenziertes Controlling- und Berichtswesen regelmäßig (monatlich) einen Überblick über die erbrachten Dienstleistungen (Produkte) und den dazu betriebenen Aufwand (Verhältnis von Kosten und Leistungen). Und so können nun Management-Entscheidungen getroffen werden, die sich effizienzsteigernd auswirken: z.B. mehr Personal in den Dienstleistungsbereichen, die zu den Kernaufgaben gehören oder mehr Sachkosten dort, wo über den Einsatz von Technik optimiert werden kann, oder weniger Personal dort, wo Aufgaben auf Dritte übertragen werden können. Die bisherigen Erfahrungen zeigen, dass so vor Ort tatsächlich Rationalisierungsgewinne möglich sind - die Gestaltungsmöglichkeiten werden größer; die Motivation, Verantwortung zu übernehmen und zu praktizieren, steigt an.

Aber, und darauf haben Autoren wie Rosemarie Barbaret, Ramon y Cayal, Hans-Ernst Böttcher, Arno Pilgram und Wolfgang Stangl immer wieder hingewiesen, diese systemimmanente Rationalisierungspolitik reicht nicht aus, die substantiellen Zukunftsfragen anzugehen und schon gar nicht sie zu beantworten. Outputorientierte Steuerung beschäftigt sich nicht mit den mittel- und langfristigen Wirkungen der Interventionen von Richtern, Staatsanwälten, Vollzugsmitarbeitern. Die Kosten- und Leistungsrechnung beschränkt sich auf Aussagen zur Effizienz, sie trifft keine Feststellungen zur fachlichen Effektivität, zur Qualität der erbrachten Dienstleistungen und $\mathrm{zu}$ ihren Folgewirkungen in den Lebenszusammenhängen der betroffenen Menschen (Tätern wie Opfern).

Im Konzept des New Public Managements wird diese Wirkungsdimension mit dem Begriff des »outcome" verbunden, also den kausal zurechenbaren Änderungen, die oft erst Wochen oder Monate nach der Intervention feststellbar sind. Arno Pilgram und Wolfgang Stangl ist zuzustimmen, dass hier ein großer Mangel an Daten, Kennzahlen, fortlaufenden Beobachtungen und Analysen festzustellen ist. Fast könnte man auf den Verdacht kommen, dass die Organe der Strafverfolgung und Strafvollstreckung sich systematisch vor einer strukturellen Feedback-Struktur schützen, weil mittel- und langfristig angelegte Wirkungsanalysen möglicherweise grundlegende Zweifel z.B. an der Resozialisierungswirkung begründen würden.

Jedenfalls haben derartige Wirkungsanalysen, die über die Zuständigkeitsgrenzen der Institutionen hinausgehen und den gesamten »Wertschöpfungsprozess« einer Dienstleistungskette betrachten, wichtige Ergebnisse erbracht: z.B. Nachuntersuchungen über die verminderte Wirkung von Anti-Aggressions-Trainings über die Zeit der Entlassung hinaus, über die verbesserte Wirkung von nach der Entlassung fortgesetzten Berufs-/ Ausbildungsprogrammen und über geringere Rückfallquoten nach sozialtherapeutischen Behandlungsmaßnahmen.

Ein wirkungsorientierter Politikansatz wird gebraucht, der hier in den nächsten Jahren Schwerpunkte setzt: Förderung der Entwicklung von Evaluierungs- und Controlling-Instrumenten, die für die Dienstleistungen von Gerichthelfern, Staatsanwälten, Richtern, Bewährungshelfern und Vollzugsmitarbeitern Fall- und Verlaufsdokumentationen und Aussagen zur Qualität und Wirkung ermöglichen. Die bisher von den Verwaltungsmodernisierern zur Verfügung gestellten Methoden reichen dazu nicht aus - hier ist die Wissenschaft im Sinne einer »Wirkungsorientierten Kriminologie« gefordert.

Das Denken in Wirkungszusammenhängen und in Prozessketten ist auch geeignet, zu einem veränderten professionellen Selbstverständnis zu kommen und nicht nur die eigene Unabhängigkeit zu betonen. Wir brauchen das Verständnis eines Kontinuums aller Interventionsformen, koordinierte Strategien, Monitoring und Evaluation als selbstverständliche professionelle Handlungsmuster und eine fortlaufende interdisziplinäre Aus- und Fortbildung.

Von der Rationalisierung der Justiz zu einer neuen Rationalität - wo sind die Promotoren, wer sind die Bündnispartner? 